\title{
Sit-To-Stand (STS) timing among older adults in their homes: Two measurement approaches
}

\author{
T.L. Grant PhD ${ }^{a, b}$ \\ M. Bryanton $\mathrm{MSC}^{\mathrm{C}}$ \\ M. Taylor MSc ${ }^{d}$ \\ H. Sveistrup $\mathrm{PhD}^{\mathrm{b}, \mathrm{c}}$ \\ M. Bilodeau PhD ${ }^{a, b, c}$ \\ F. Knoefel MD ${ }^{\mathrm{a}, \mathrm{d}, \mathrm{e}}$ \\ R. Goubran $\mathrm{PhD}^{\mathrm{c}}$ \\ J. Jutai $\mathrm{PhD}^{f}$
}

\begin{abstract}
${ }^{a}$ Bruyère Research Institute, Ottawa, Canada; ${ }^{b}$ School of Rehabilitation Sciences, University of Ottawa, Ottawa, E: TGrant@bruyere.org; 'School of Human Kinetics, University of Ottawa, Ottawa, E: MBryanton@bruyere.org; ${ }^{d}$ Department of Systems and Computer Engineering, Carleton University, Ottawa; 'Department of Community and Family Medicine, University of Ottawa, Ottawa; Interdisciplinary School of Health Sciences, University of Ottawa, Ottawa ON, Canada
\end{abstract}

T.L. Grant, M. Bryanton, M. Taylor, H. Sveistrup, M. Bilodeau, F. Knoefel, R. Goubran, J. Jutai, Sit-to-stand (STS) timing among older adults in their homes: Two measurement approaches. Gerontechnology 2014;12(3):153-158; doi:10.4017/gt.2014.12.3.006.00 Sit-to-stand (STS) times required by older adults exiting from their beds at home were compared simultaneously using under mattress pressure sensor technology and video analysis. Six older adults consented to participate in the study and had under mattress pressure technology installed in their homes. Participants were visited monthly over a period of 2-10 months by a researcher who asked them to perform three bed exits which were videotaped. The pressure sensor technology calculated a portion of STS time based on changes in pressure between the end of stable sitting on the edge of the bed and pressure leaving the bed. The video analysis approach calculated this corresponding initial phase of the same STS transfer by determining the difference between the first forward movement from the quiet sitting position and the initiation of vertical rise. The remaining period of vertical rise into a complete standing position was calculated using video analysis but not pressure sensor technology. A Spearman's rank order correlation analysis revealed that there was a moderate, positive correlation between the video and pressure-sensor measures of the initial phase of STS which was statistically significant $\left(r_{s}=0.50, n=97, p<0.001\right)$. Wilcoxon signed-rank tests indicated that pressure sensor times were longer $(Z=-7.91, p<0.000)$ and more variable than video times $(Z=-3.14, p=0.002)$. Analysis of video data revealed that this initial phase of STS represented between 15 to $81 \%$ of the total STS time among the 97 sampled STS in this study. Results highlight how the home environment presents a number of conditions affecting differences in STS values obtained by each of these measurement approaches.

\section{Keywords: home monitoring, under mattress pressure sensors, sit-to-stand, aging}

The ability to rise from a sitting position is a fundamental activity of daily living and one that supports independent living as individuals grow older. Individuals who have slower sit-to-stand (STS) times are more likely to have balance disorders ${ }^{1}$ and greater deficits in instrumental activities of daily living ${ }^{2}$. Difficulty with this common activity differentiates healthy older adults from those who fall $^{3}$ as well as from those who live in institutions ${ }^{4}$.

Literature on STS commonly identifies several phases of the movement. Key commonalities of various classification schemes include the horizontal movement of center of mass in preparation for the vertical rise from a relatively large base of support to a more narrow base of support in the standing position ${ }^{5}$. Changes in the control and duration of center of mass movement during the first horizontal phase of STS have been associated with declines in strength and postural control in older adults ${ }^{6-8}$. With advancing age, individuals take longer to bring their center of mass forward to a more anterior position before initiating the vertical or "seat off" phase of the STS 
compared to younger individuals ${ }^{9}$. This tendency is even greater in frail older adults who have less ability to use a vertical lift off strategy and therefore compensate with a forward flexion strategy ${ }^{10}$.

Changes in this initial phase of the STS transfer may indicate the onset of frailty and if detected early enough may assist in the prevention of mobility decline. One such tool for monitoring this phase of the STS transfer on a continual basis is under-mattress pressure technology. This type of technology is placed under a bed mattress and uses pressure change to calculate STS time ${ }^{11-13}$. We have previously shown that under-mattress pressure sensors can differentiate between healthy older adults and those with mobility impairments on the basis of STS time in the laboratory environment $^{11}$. In this experiment, times required to stand from a bed were similar to times required to stand from a chair for both younger ${ }^{14}$, older ${ }^{15,16}$ and mobility impaired adults ${ }^{15,16}$. Although laboratory data suggests that under-mattress pressure sensors have promise as a long-term monitoring tool, no research has examined the use of this technology for monitoring STS timing in the home context.

A commonly used form of criterion measurement is video recording followed by computer assisted motion analyses $5,14,17$. Video analysis provides a level of confidence to the researcher in being able to view contextual information in relation to the movement being performed. In other words, one can view how the task is being performed in relation to environmental variables which may explain a change in performance. Video analysis also can provide information on the later phase of the STS movement not captured by pressure sensors - namely the phase occurring after pressure from hands and buttocks have left the bed. The drawback of video however, is that it raises privacy concerns and therefore may be less acceptable to older adults ${ }^{18,19}$.

As the development of new technologies like under-mattress sensors progresses, it is important to test them in the home environment for comparison with previously used reference measures such as video analysis. Although video and pressure measures have been compared in laboratory $^{12}$, they have not been compared in the home environment. The importance of collecting data in the home environment is that it provides insights on how tasks like sit-to-stand can be affected by context. Understanding the role of context on measurement correlation is critical to the development of useful home monitoring systems. Therefore, this paper compares the measurement of the initial phase of sit-to-stand (STS) timing obtained from under-mattress sensors with that obtained using video motion analysis in the home environment of older adults. We hypothesize that STS measures obtained by these two technologies will correlate significantly.

\section{Methods \\ Participants}

Six older adults (5 female) living independently in the community consented to participate in this study which had been approved by the Research and Ethics Boards of Bruyère Continuing Care (Ottawa, Ontario, Canada) and the University of Ottawa. The study was conducted within the context of a larger research project examining bed mobility among older adults. Participant mean age was $77 \pm 9$ years. All were able to rise from bed independently. Two individuals used a bed rail for assistance to exit their beds. The participants represented a sample of convenience.

\section{Under-mattress sensors}

The under-mattress sensors were manufactured by S4 Sensors Inc. (Victoria, British Columbia, Canada). The technology consisted of fiber optic pressure sensors embedded in a mat with the dimensions of $860 \times 860 \mathrm{~mm}$. Each pressure-sensitive mat had 72 equally spaced sensors in an $8 \times 9$ grid array. The pressure-sensitive mat was connected to a transmitter box which conveyed pressure data to a Dell OptiPlex PC via a Bluetooth connection. The pressure-sensitive mat was placed under the participant mattress while the computer box was placed under the bed.

\section{Pressure sensor processing}

A processing algorithm was developed in MAT$\mathrm{LAB}$ version 7.12 (MathWorks, Inc. Massachusettes USA) to identify when bed exits occurred within the large sensor data set and is described in an earlier publication ${ }^{20}$. For the purposes of this study, the STS portion of the bed exit was calculated individually by viewing a processed pressure tracing in MATLAB. The end of the STS was defined by the absence of pressure on the pressure mat. The beginning of STS was defined as the time when peak pressure on the mat was recorded prior to the end of the STS. The difference of the peak sitting pressure and the absence of pressure was considered to be STS time as measured by the mat. This timing measure reflected the time it took for the participants' last point of contact to leave the bed from a stable sitting position on the edge of the bed. The measure did not include the time it took participants to rise into sitting from the lying position.

\section{Performance of STS from bed}

Participants were visited monthly by a researcher and asked to perform three bed transfers. The transfers were also recorded by a single hand held video camera from a side angle. A single 
camera side angle view was employed since consistent space restrictions in the home context did not permit a double tripod set up to capture both front and side views. Instructions to participants were: "Please get out of bed as you normally do in the morning". These instructions were intended to be simple and to produce a picture of what might happen in unobserved situations of daily routine. Time and date of video recordings were documented so that the same transfers could be analyzed from the information recorded by the pressure sensors. Participants were asked to perform three bed exits consecutively during the researcher visit if this was within their physical capacity.

\section{Video analysis}

Video recordings of each bed exit were uploaded onto a computer, where Dartfish Connect ${ }^{\mathrm{TM}}$ version 6.0 (Dartfish Connect ${ }^{\mathrm{TM}}$, Lausanne, Switzerland) was used for video STS time analyses. This software allowed the researcher to view each video clip of the observed bed exit at several speeds, as well as to advance by a single frame in order to identify critical time points during each bed exit.

The total video sit-to-stand (vSTS-total) time was broken into two parts; the 'preparation start' (vSTS-1) phase and the 'ascension' (vSTS-2) phase. vSTS-1 was defined as the period between the time at which forward momentum began (angle increases between the vertical and the head/ trunk) until the moment of weight off or ascension. vSTS-2 was defined as the period between when the participant's buttocks left the mattress and full upright posture (hips and knees fully extended with the back in a vertical position).

Once critical time points were determined, vSTS1 and vSTS-2 were calculated using Excel ${ }^{\mathrm{TM}}$ version 14.0 (Microsoft Inc., Washington, USA), as the change in time between start and end time points of each phase.

This paper focuses on reporting the STS- 1 time as measure via video analysis (vSTS-1) in relation to the (corresponding) STS-1 time measured by the pressure-sensitive mat technology (pSTS-1). In addition to this relationship, we also calculated the percent (\%) of vSTS-total that vSTS-1 represented. This was done in order to provide some indication of the portion of the bed exit that would not be captured by under mattress pressure sensors. The $\%$ of vSTS-total represented by vSTS-1 was calculated for each participant for each visit.

\section{Observation of video recordings}

The timing of vSTS- 1 and vSTS- 2 was calculated by two researchers who also made descriptive notes after viewing the video recordings (TLG,
$M B)$. The notes were used to identify atypical movement patterns and ways in which the home context may have affected STS performance. The purpose was to supplement the timing information and assist with interpretation of potential differences in timing measures between video and pressure mat results.

\section{Comparison of approaches}

Statistical analyses were performed using the SPSS Statistics software package, version 21.0 (IBM Corp. Armonk, NY, USA). Normality of the data was tested and non-parametric measures selected to compare measurement approaches. A Wilcoxon signed-ranks test was used to compare: (i) the vSTS- 1 and pSTS- 1 means, and (ii) the inter-trial variability of each measurement approach. The linear relationship between vSTS1 and pSTS-1 was determined using a Spearman's rank order correlation analysis.

\section{RESULTS}

A total of 97 sit-to-stand times were obtained from the six participants using each measurement method (i.e. video analysis and pressure sensor technology). The mean vSTS- 1 was 0.74 $( \pm 0.53)$ seconds while the mean pSTS- 1 was 1.28 $( \pm 0.64)$ seconds. These two means were significantly different $(Z=-7.91, p<0.000)$. The pSTS-1 times were longer than vSTS- 1 in 86 out of the 97 transfers. Figure 1 displays box and whisker plots showing median, interquartile range, minimum and maximum STS values obtained by video and pressure senors for each participant measured in seconds.

There was a statistically significant difference in the inter-trial variability between the two measures with pSTS-1 being more variable than vSTS- 1 in 17 out of 20 cases $(Z=-3.14, p=0.002)$. Inter-trial variability could only be conducted on visit trials during which participants felt they were capable of repeating more than one bed exit consecutively.

There was a moderate positive correlation between the video and pressure-sensor measures which was statistically significant, $r_{s}=0.50, n=97$, $\mathrm{p}<0.001$ (Figure 2).

Notes taken on visual observations of transfer patterns are summarized in Table 1. These observations represented cases where (i) a participant performed the bed exit in a way that differed from their usual pattern or (ii) context-specific information from the home environment would have influenced under-mattress pressure mat data but would not have been apparent in the dataset. Observations were grouped in related categories with the intention of providing insight 


\section{Sit-to-Stand timing}

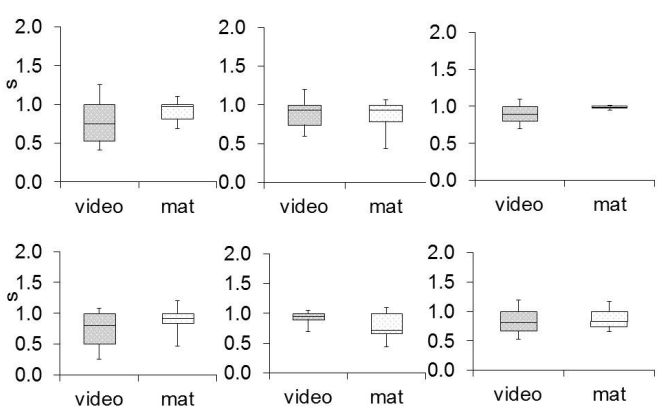

Figure 1. Box and whisker plots of STS values obtained by video and pressure sensors (mat) for each participant

into potential timing discrepencies resulting from the environmental context.

Amoung our sample of 6 participants, the time used for vSTS-1 ranged from between 15 to $81 \%$ of the total vSTS time (Figure 3). As shown, a significant percentage of the total vSTS time occured in the vSTS-2 phase. Mean \%vSTS-total is shown in Figure 3 with an icon while the minimum and maximum ranges are represented with error bars.

\section{Discussion}

The results obtained in this study indicate that STS1 times obtained from pressure sensors are longer and more variable than those obtained by video analysis in the home context. Despite these differences, the two measurement methods are moderately but significantly correlated. This latter finding supports our original hypothesis and offers promise that under mattress pressure sensors can play a role in monitoring changes that occur over time among frail older adults in the home context. The home context presents a number of differences from highly controlled laboratory situations where correlations have been found to be higher ${ }^{11,12}$. This study has identified some of these particularities which are important to recognize for the development of viable home monitoring systems.

Our observations highlighted some common reasons why pressure sensor times are generally longer than video times such as the adjustment of slippers or leaning on the bed after standing.

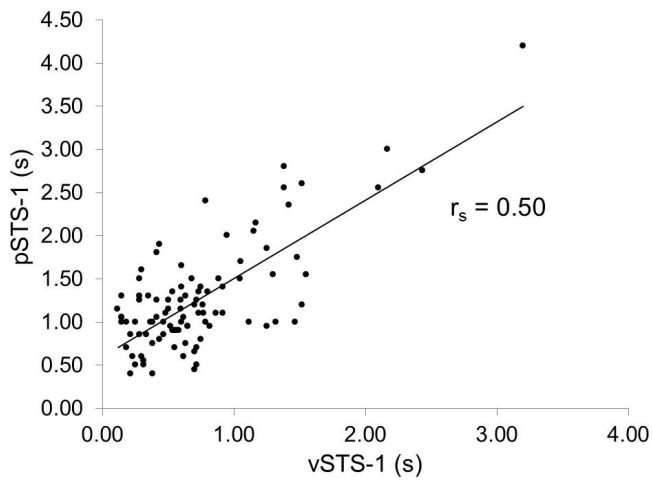

Figure 2. Correlation of pressure sensor (pSTS-1) and video (vSTS-1) Sit-To-Stand time values
Higher inter-trial variablity observed in the pressure sensor values can be accounted for due to similar reasons noted in our observation table. The greater inter-trial variablity also indicates that under mattress pressure sensors are highly sensitive to changes in transfer performance and therefore offer the potential to capture rich information on bed exit performance relevant to pressure change.

The key to using this sensitive information in a way that is clinically relevant will involve developing methods to differentiate whether longer pressure sensor transfer times are related to a change in functional status or simply reflective of day-to-day variability. For example, it may be that a participant starts to lean on the bed after exiting with increasing frequency because of increasingly impaired balance. The decrease in function would need to be differentiated from a situation where the user was simply anticipating re-entry into the bed. A key implication of our findings is that timing values obtained from pressure sensor technology will need to be interpreted in the context of distinct patterns over time and most likely in combination with other variables related to the STS movement. These variables could include time spent in bed $^{20}$ or the presence of movement patterns such as bouncing when preparing to exit the bed, which has been associated with mobility difficulties ${ }^{21}$.

Our results also indicated that there is a substantial portion of STS which cannot be monitored

Table 1. Some observed bed-exit patterns

\begin{tabular}{l|l|l}
\hline Hand placement to stand-up & Sliding action & Extraneous activity \\
\hline $\begin{array}{l}\text { Uses bedrail } \\
\text { Leaves hand on bed after exit }\end{array}$ & $\begin{array}{l}\text { Slides off edge of bed } \\
\text { Slides off edge of bed and maintains } \\
\text { contact with bed in upright position } \\
\text { Slides towards head of bed before } \\
\text { standing up }\end{array}$ & $\begin{array}{l}\text { Dons slippers in seated position } \\
\text { Places dog on floor before standing- } \\
\text { up } \\
\text { Dog stays on bed }\end{array}$ \\
$\begin{array}{l}\text { Places hand on night } \\
\text { table/dresser }\end{array}$ & & \\
\hline
\end{tabular}




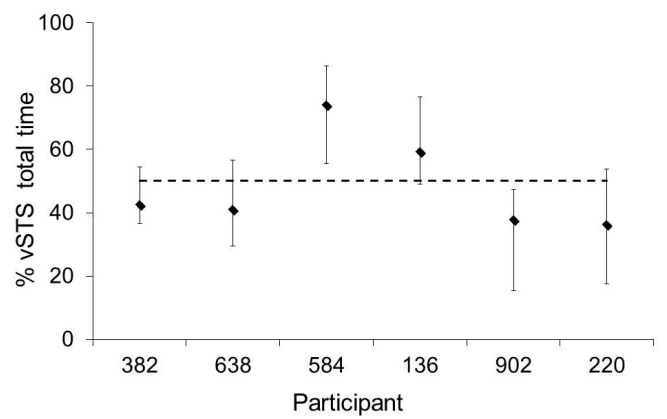

Figure 3. Percent total vSTS (Video Time-To-Stand) value represented by vSTS-1; mean \%STS total is shown with an icon

by under-mattress pressure sensors and that this portion is highly variable. The vSTS-1 value in this study was as low as $15 \%$ and as high as $81 \%$ of vSTS-total. In cases where a hand was left on the bed for stability, this portion of the transfer would obviously be higher than if the hands were held to the side or placed on a different surface than the bed. The increasing dependence of hands during bed exit has been associated with greater difficulty in performing STS ${ }^{5}$ and therefore could be an important focus of sensor development. In the current study, participants used their hands on a variety of surfaces including bed rails and night stands to rise. These observations indicate that monitoring hand pressures on multiple surfaces around the bed will be important in order to determine whether there is an indication of increasing hand/upper extremity use. Furture development of remote mobility monitoring in the home environment could incorporate sensor technology to capture hand use on assistive devices including bedrails and nightstands, common structures around the bed that are used during bed exits and entries. Of equal importance, incorporating floor sensors will permit determination of pressure patterns during the second phase of STS and facilitate identification of balance specific changes in performance of the transfer.

We observed that the vSTS-1 represented a highly variable percentage of the total vSTS transfer time

\section{Acknowledgements}

This study was supported by grants from the Canadian Institutes for Health Research (CIHR) and National Sciences and Engineering Research Council of Canada (NSERC). We would also like to thank our participants for volunteering, Melissa Donskov for her managerial support and Amy Porteus, the project's knowledge user.

\section{References}

1. Thapa PB, Gideon P, Fought RL. Comparison of clinical and biomechanical measures of balance and mobility in elderly nursing home residents. both within and between participants. While previous literature suggests that spending more time in the intial forward phase of STS is related to advancing age and frailty ${ }^{7,8,10}$, our observations illustrate how the home environment may modify this relationship. For example, one atypical pattern observed was an individual, with a relatively high bed, who slid off of the bed and maintained contact with the bed once in the standing position. In this example, the individual substituted the forward flexion strategy with a stabilize and slide strategy. Thus, instead of gaining greater postural control by prolonging the initial phase of the transfer, an individual can initiate the vertical phase earlier by stabilizing their legs against the bed and allowing gravity to assist with forward movement. The importance of this observation is that there are multiple strategies for performance of STS in the home context and this variability is important to consider in the development of home monitoring systems. These strategies may differ according to whether an individual chooses to get up quickly to go to the washroom or slowly due to pain or relative bed height. A critical factor in developing home monitoring systems will be to characterize the frequency of patterns used by any one individual in combination with other characterstics of bed mobility. This information will permit the identification of markers that characterize important functional changes as opposed to day-to-day variablity.

\section{Conclusion}

Sit-to-stand times determined with under-mattress pressure sensors were longer and more variable than those obtained with visual video analysis. These measurement approaches were moderately, significantly and positively correlated suggesting that both methods detected similar aspects of the STS task. The home environment presents a number of conditions that influence STS times which differ from controlled laboratory enviroments. These conditions will be important to consider in the future development sensor algorithms for remote monitoring of STS and the potential to use the information for identification of functional changes in older adults.

\section{Journal of the American Geriatrics Society} 1994;42(5):493-500

2. Judge JO, Schechtman K, Cress E. The relationship between physical performance measures and independence in instrumental activities of daily living. Journal of the American Geriatrics Society 1996;44(11):1332-1341

3. Doheny EP, Chie WF, Foran T, Greene BR, Cunningham C, Kenny RA. An instrumented sit-tostand test used to examine differences between older fallers and non-fallers. Engineering in Medicine and Biology Society, Annual International Conference of the IEEE. Engineering in Medi- 
cine and Biology Society 2011; pp 3063-3066; doi:10.1109/IEMBS.2011.6090837

4. Leon J, Lair T. Functional status of the non-institutionalized elderly: estimates of ADL and IADL difficulties. Rockville: Agency for Health Care Policy and Research. Report No. PHS 90-3462; 1990

5. Janssen WGM, Bussmann HBJ, Stam HJ. Determinants of the sit-to-stand movement: a review. Physical Therapy 2002 Sep;82(9):866-879

6. Hughes MA, Schenkman ML. Chair rise strategy in the functionally impaired elderly. Journal of Rehabilitation Research and Development 1996;33(4):409-412

7. Mourey F, Grishin A, D'Athis P, Pozzo T, Stapley P. Standing up from a chair as a dynamic equilibrium task: A comparison between young and elderly subjects. Journals of Gerontology Series A 2000;55(9):B425-B431; doi: 10.1093/ gerona/55.9.b425

8. Schultz AB, Alexander NB, Ashton-Miller JA. Biomechanical analyses of rising from a chair. Journal of Biomechanics 1992;25(12):1383-1391; doi:10.1016/0021-9290(92)90052-3

9. Alexander NB, Schultz AB, Warwick DN. Rising from a chair: effects of age and functional ability on performance biomechanics. Journal of Gerontology 1991;46(3):M91-M98; doi:10.1093/ geronj/46.3.m91

10. Doorenbosch CAM. Two strategies of transferring from sit-to-stand; the activation of monoarticular and biarticular muscles. Journal of Biomechanics 1994;27(11):1299-1307; doi:10.1016/00219290(94)90039-6

11. Arcelus A, Herry CL, Goubran RA, Knoefel F, Sveistrup H, Bilodeau M. Determination of Sit-toStand Transfer Duration Using Bed and Floor Pressure Sequences. IEEE Transactions on Biomedical Engineering 2009;56(10):2485-2492; doi:10.1109/ TBME.2009.2026733

12. Arcelus A, Veledar I, Goubran R, Knoefel F, Sveistrup H, Bilodeau M. Measurements of Sit-toStand Timing and Symmetry From Bed Pressure Sensors. IEEE Transactions on Instrumentation and Measurement 2011;60(5):1732-1740; doi:10.1109/ TIM.2010.2089171

13. Veledar I, Arcelus A, Goubran R, Knoefel F, Sveistrup H, Bilodeau M. Sit-to-stand timing measurements using pressure sensitive technology. Instrumentation and Measurement Technology Conference (I2MTC); 2010 IEEE; pp 1337-1340;
doi:10.1109/IMTC.2010.5488285

14. Goffredo M, Schmid M, Conforto S, Carli M, Neri A, D'Alessio T. Markerless human motion analysis in Gauss-Laguerre transform domain: An application to sit-to-stand in young and elderly people. IEEE Transactions on Information Technology in Biomedicine 2009;13(2):207-216; doi:10.1109/ TITB.2008.2007960

15. Najafi B, Aminian K, Loew F, Blanc Y, Robert PA. Measurement of stand-sit and sit-stand transitions using a miniature gyroscope and its application in fall risk evaluation in the elderly. IEEE Transactions on Biomedical Engineering 2002;49(8):843-851; doi:10.1109/TBME.2002.800763

16. Salarian A, Russmann H, Vingerhoets FJ, Burkhard PR, Aminian K. Ambulatory monitoring of physical activities in patients with Parkinson's disease. IEEE Transactions on 2007;54(12):2296-2299; doi:10.1109/TBME.2007.896591

17. Fotoohabadi MR, Tully EA, Galea MP. Kinematics of rising from a chair: image-based analysis of the sagittal hip-spine movement pattern in elderly people who are healthy. Physical Therapy 2010;90(4):561-571; doi:10.2522/ptj.20090093

18. Demiris G, Parker OD, Giger J, Skubic M, Rantz R. Older adults' privacy considerations for vision based recognition methods of eldercare applications. Technology and Health Care 2009;17(1):4148; doi:10.3233/THC-2009-0530

19. Townsend D, Knoefel F, Goubran R. Privacy versus autonomy: A tradeoff model for smart home monitoring technologies. Proceedings of the Annual International Conference of the IEEE Engineering in Medicine and Biology Society 2011; pp :4749-4752; doi:10.1109/IEMBS.2011.6091176

20. Taylor M, Grant T, Knoefel F, Goubran R. Bed occupancy measurements using under mattress pressure sensors for long term monitoring of community-dwelling older adults. IEEE International Symposium on Medical Measurements and Applications (MeMeA) 2013; pp 130-134; doi:10.1109/MeMeA.2013.6549721

21. Arcelus A, Goubran R, Knoefel F, Sveistrup H, Bilodeau M. Detection of bouncing during sit-tostand transfers with sequential pressure images. Proceedings of the IEEE International Workshop on Medical Measurements and Applications (MeMeA) 2011; pp 158-161; doi:10.1109/MeMeA.2011.5966665 